\title{
OXYGEN UPTAKE KINETICS IN TRAINED ADOLESCENT FEMALES
}

Viswanath B. Unnithan ${ }^{1}$, Denise M. Roche ${ }^{2}$, Max Garrard ${ }^{3}$, Kathryn Holloway ${ }^{2}$ and Simon Marwood ${ }^{2}$

${ }^{1}$ Centre for Sport, Health and Exercise Research, Staffordshire University, Stoke-onTrent, UK, ${ }^{2}$ Department of Health Sciences, Liverpool Hope University, Liverpool, UK, ${ }^{3}$ Active Lifestyles Research Centre, Carnegie Faculty, Leeds Metropolitan University, Leeds, UK

Address for correspondence:

Professor V.B. Unnithan

Centre for Sport, Health and Exercise Research

Faculty of Health Sciences

Staffordshire University

Brindley Building

Leek Road

Stoke-on-Trent

ST4 2DF

UK

Tel: 01782295986

E-mail: V.Unnithan@staffs.ac.uk

FAX: 01782294321

Running Title: Oxygen uptake kinetics in young athletes 


\begin{abstract}
Little evidence exists with regard to the effect that exercise training has upon oxygen uptake kinetics in adolescent females. PURPOSE: The aim of the study was to compare $\dot{V}_{2}$ and muscle deoxygenation kinetics in a group of trained (Tr) and untrained (Utr) female adolescents. METHOD: Twelve trained (6.4 \pm 0.9 years training, $10.3 \pm 1.4$ months per year training, $5.2 \pm 2.0$ hours per week) adolescent female soccer players (Age: $14.6 \pm 0.7$ years) were compared to a group $(n=8)$ of recreationally active adolescent girls (Age: $15.1 \pm 0.6$ years) of similar maturity status. Subjects underwent two, 6-min exercise transitions at a workload equivalent to $80 \%$ of lactate threshold from a 3-min baseline of 10W. All subjects had a passive rest period of 1 hour between each square-wave transition. Breath-by-breath oxygen uptake and muscle deoxygenation were measured throughout and were modelled via a monoexponential decay with a delay relative to the start of exercise. RESULT: Peak $\dot{V}_{2}$ was significantly $(\mathrm{p}<0.05)$ greater in the Tr compared to the Utr (Tr: $43.2 \pm 3.2 \mathrm{~mL} \cdot \mathrm{kg}^{-1} \mathrm{~min}^{-1}$ vs. Utr: $34.6 \pm 4.0 \mathrm{~mL} \cdot \mathrm{kg}^{-}$ $\left.{ }^{1} \mathrm{~min}^{-1}\right)$. The $\dot{V}_{2}$ time constant was significantly $(\mathrm{p}<0.05)$ faster in the Tr compared to the Utr (Tr: $26.3 \pm 6.9$ s vs. Utr: $35.1 \pm 11.5$ s). There was no inter-group difference in the time constant for muscle deoxygenation kinetics (Tr: $8.5 \pm 3.0 \mathrm{~s}$ vs. Utr: $12.4 \pm 8.3 \mathrm{~s}$ ); a large effect size, however, was demonstrated (-0.804). CONCLUSION: Exercise training and/or genetic self-selection results in faster kinetics in trained adolescent females. The faster $\dot{V} \mathrm{O}_{2}$ kinetics seen in the trained group may result from enhanced muscle oxygen utilisation.
\end{abstract}

Key Words: fundamental phase, deoxygenation kinetics, youth athletes, moderatedomain exercise 


\begin{tabular}{|c|c|}
\hline \multicolumn{2}{|c|}{ Abbreviations } \\
\hline$A_{\mathrm{Vo}_{2}}$ & The asymptotic amplitude of the oxygen uptake fundamental \\
\hline & (phase II) response \\
\hline$A_{H H b}$ & The asymptotic amplitude of the deoxyhaemoglobin response, \\
\hline $\mathrm{Db}$ & Body density \\
\hline FFM & Fat free mass \\
\hline $\mathrm{HbO}_{2}$ & Muscle oxyhaemoglobin concentration \\
\hline $\mathrm{HHb}$ & Deoxyhaemoglobin concentration \\
\hline \multirow{2}{*}{$H H b_{(b)}$} & Mean deoxyhaemoglobin response measured during the final \\
\hline & minute of baseline \\
\hline HR & Heart Rate \\
\hline MRT & Mean response time \\
\hline NIRS & Near-infrared spectroscopy \\
\hline Peak $\dot{V}_{2}$ & Peak oxygen uptake \\
\hline $\mathrm{p} \dot{V}_{O_{2}}$ & Pulmonary oxygen uptake kinetics \\
\hline RER & Respiratory exchange ratio \\
\hline$T D_{V_{o_{2}}}$ & Time delay for oxygen uptake relative to the onset of exercise \\
\hline \multirow[t]{2}{*}{$\tau_{\mathrm{Vo}_{2}}$} & Time constant for oxygen uptake for the fundamental component \\
\hline & of the response \\
\hline \multirow[t]{2}{*}{$T D_{\mathrm{HHb}}$} & Time delay for deoxyhaemoglobin concentration relative to the \\
\hline & onset of exercise \\
\hline$\tau_{H H b}$ & Time constant for deoxyhaemoglobin. \\
\hline $\mathrm{THb}$ & Total haemoglobin concentration \\
\hline $\mathrm{V}_{\mathrm{E}}$ & Pulmonary ventilation \\
\hline $\mathrm{VO}_{2}$ & oxygen uptake \\
\hline$\dot{V} O_{2(S S)}$ & Steady state oxygen uptake \\
\hline$\dot{V} O_{2(b)}$ & Mean oxygen uptake measured during the final minute of baseline \\
\hline $\mathrm{VCO}_{2}$ & Volume of carbon dioxide produced \\
\hline Yo-Yo IR1 & Intermittent recovery Test 1 \\
\hline Yo-Yo IR2 & Intermittent recovery Test 2 \\
\hline
\end{tabular}




\section{Introduction}

Pulmonary oxygen uptake $\left(\mathrm{p} \dot{V}_{2}\right)$ kinetics provides a surrogate measure of the body's ability to transport and utilise oxygen at the muscle level during the transition from rest to exercise (Fawkner and Armstrong, 2003). Furthermore, evaluating the time-course of oxygen uptake changes following the onset of exercise also provides an indirect estimate of the contribution of non-aerobic energy sources to this type of activity (Potter and Unnithan 2005). Perhaps the most potent stimulus for speeding $\mathrm{p} \dot{\mathrm{V}} \mathrm{O}_{2}$ kinetics, as measured by a reduction in time constant of the fundamental phase, and thus indicating a reduction in the contribution of non-aerobic energy sources, is exercise training. There are multiple examples from the scientific literature (e.g. Bailey et al. 2009; Berger et al. 2006; Jones and Koppo 2005; Koppo et al. 2004) of exercise training resulting in a reduction in the time constant of the fundamental phase of $\mathrm{p} \dot{\mathrm{VO}}_{2}$ kinetics at both moderate and heavy exercise intensities in adult populations

The influence of training on the oxygen uptake kinetic response in children and adolescents is less well characterized. Earlier evidence from cross-sectional studies seemed to indicate that exercise training had no effect in reducing the time constant of the fundamental phase of $\mathrm{p} \dot{V_{2}}$ kinetics in pre-pubertal children (Cleuziou et al. 2002; Obert et al. 2000). More recent cross-sectional studies, however, have demonstrated that systematic exercise training may result in a speeding of the fundamental phase of $\mathrm{p} \dot{V} o_{2}$ kinetics in both children (Winlove et al. 2010) and adolescents (Marwood et al. 2010, 
McNarry et al. 2011). Such discrepancies may be explained by the lack of congruence between the training and experimental exercise modality in the earlier studies (Cleuziou et al. 2002; Obert et al. 2000). However, the paucity of data characterizing p $\dot{\mathrm{V}} \mathrm{O}_{2}$ kinetics in trained adolescents is significant since evidence exists that during maturation, the balance of oxidative and glycolytic enzyme activities may change (Haralambie et al. 1982, Peterson et al. 1999). Hence data collected in adult populations may not necessarily be transferable to adolescents. Furthermore, in all but one of the above stated studies in children and adolescents (Marwood et al., 2010), the primary training stimulus was endurance training; little data exists on the impact of high-intensity, intermittent exercise, which characterises soccer match-play, on $\mathrm{p} \dot{\mathrm{VO}}_{2}$ kinetics in the trained adolescent population

Soccer is characterised by multiple transitions from low to high intensity exercise (Buchheit et al., 2010). Since fatigue during high-intensity exercise is related to the reliance on non-aerobic energy sources, the ability to transition rapidly from rest to exercise is likely to characterise successful performers in this sport. Hence it might be expected that a history of systematic, high-intensity, intermittent exercise training which characterises soccer-play, will be associated with faster $\mathrm{p} \dot{V_{O}}$, kinetics. Indeed, a faster fundamental phase time constant derived from moderate intensity exercise, was significantly related to better Yo-Yo IR1 and IR2 soccer field test performance (Rampinini et al., 2010) and reduced time decrements in repeated sprint tests in adult male soccer players (Dupont et al., 2005). 
With increasing numbers of young females involved in soccer training and competition (Datson et al., 2014); soccer therefore represents an important model to explore the influence that high-intensity, intermittent training may have on changes in the fundamental phase of $\mathrm{p} \dot{V}_{2}$ kinetics in this population. Hence the primary purpose of the present study was to compare $\mathrm{p} \dot{V}_{2}$ kinetics during the transition to moderate intensity exercise in elite adolescent female soccer players, with,a group of maturation-matched untrained females. We hypothesised that $\mathrm{p} \dot{\mathrm{V}}_{2}$ kinetics would be faster in the trained population versus the untrained controls.

In the adolescent population, we have previously demonstrated no difference in deoxyhaemoglobin (HHb) kinetics between trained soccer players and recreationally active controls during the transition to moderate intensity exercise (Marwood et al. 2010). However, McNarry et al. (2011) demonstrated faster HHb kinetics in trained female adolescent swimmers compared to their recreationally active peers during heavy intensity exercise. The former finding indicates that the training-induced speeding of $\mathrm{p} \dot{\mathrm{O}_{2}}$ kinetics is due to an equal contribution of adaptations in oxygen delivery and utilization, whereas the latter indicates that adaptations in oxygen utilization are relatively more important. Whether these equivocal findings are due to the differences in training history or sex is presently unclear. Hence the secondary purpose of the present study was to determine $\mathrm{HHb}$ kinetics as a means of examining the mechanisms underpinning any training-induced speeding of $\mathrm{p} \dot{V} \mathrm{O}_{2}$ kinetics. 


\section{Methods:}

\section{Subjects}

Twelve trained female adolescent soccer players (age: $14.6 \pm 0.7$ years ) and eight recreationally active adolescent girls(age: $15.1 \pm 0.6$ years) volunteered to participate in this study. Maturity status was determined by Tanner's five stages of self-assessment for breast development, which has previously been validated (Matsudo and Matsudo 1994). Tanner stages for the trained and untrained groups ranged from Tanner stage 3-5. Two subjects in the trained group were unable to understand the directions for the Tanner staging and, therefore, did not complete the self-assessment correctly. Information regarding the ovulatory cycle phase of each subject was obtained via a menstrual cycle questionnaire that was completed at each visit. All participants completed a physical activity questionnaire modified from Bar-Or (1983).

The trained girls were recruited from two professional soccer clubs in the North-West of England. All the girls were engaged in regular, systematic training (6.4 \pm 0.9 years training, $10.3 \pm 1.4$ months per year training, $5.2 \pm 2.0$ hours per week, and $5.9 \pm 1.0$ years playing competitive soccer matches). The eight untrained subjects were recreationally active, but not engaged in any exercise training. Written informed consent from the parents/guardians and verbal and written assent from the subjects were obtained prior to the start of the study. The study was approved by a local University ethics committee. 


\section{Study Design:}

Subjects visited the exercise physiology laboratory twice. All subjects completed a pretest questionnaire in order to verify that they were in a post-prandial state at each visit ( 3 h), had refrained from vigorous exercise in the preceding 48 hours and had not consumed caffeinated products (i.e. soft drinks, tea, chocolate) 3 h prior to the cycle ergometry testing. Body mass and stature using stretch height were measured following the International Standards for Anthropometric Assessment (2001). Body mass (nearest $0.1 \mathrm{~kg}$ ) was measured in kilograms on a calibrated electronic scale (BodPod, Life Measurement Instruments, Concord, CA). Stretch height was measured in centimeters using a wall-mounted stadiometer. Air displacement plethysmography (Bodpod, Concord, CA) was used to determine body density (Db). Fat mass and fat-free mass (FFM) were then determined using the following formula, $\%$ fat $=(5.07 / \mathrm{Db}-4.64) \cdot 100$ for females (Table 1). The air displacement plethysmography measure of body composition has been demonstrated to be valid in both adults and children (Demerath et al. 2002).

At the first visit, all subjects performed an incremental cycle ergometer (Lode Excalibur Sport, Groningen, The Netherlands) test to volitional exhaustion. The protocol consisted of 3-min stages, with increments of 35W, starting with an initial workload of 35W. Subjects maintained a cadence of 60rpm throughout the tests. Physiological criteria for achievement of peak exercise were a heart rate within 10 beats of 200 beats $\cdot \mathrm{min}^{-1}$ and a respiratory exchange ratio (RER) of $\geq 1$. Peak oxygen uptake was defined as the mean 
of the two highest 30-s values during the final exercise increment (Table 1). Lactate threshold was determined using the standard v-slope method and visual inspection of the ventilatory equivalent and end-tidal pressure plots for oxygen and carbon dioxide respectively (Burnley et al. 2006). The visual inspection of the plots was conducted independently by two of the research team. At the second visit, all subjects completed two 6-min square wave transitions from a 3-min baseline of $10 \mathrm{~W}$ to $80 \%$ of the workload at which the gas exchange threshold was achieved. Each transition was separated by a minimum of 1 hour of passive rest.

\section{Measurements:}

The Cosmed K4b² (Rome, Italy) was used to obtain cardio-respiratory measurements of: $\mathrm{V}_{\mathrm{E}}, \dot{\mathrm{V}}_{2}, \mathrm{VCO}_{2}$ and RER. Calibration of the $\mathrm{O}_{2}$ and $\mathrm{CO}_{2}$ sensors were performed prior to each test trial, with standard gases of known oxygen (16\%) and carbon dioxide (5\%) concentrations. A 3-liter volume calibration syringe was used to calibrate respiratory volume. Resting and exercise data were calculated on a breath-by-breath basis. These data were subsequently averaged over 20-s epochs to determine submaximal steady state and peak values. Heart Rate (HR) was measured using short-range telemetric techniques (Polar S610, Kempele, Finland).

During the square-wave exercise bouts, continuous non-invasive measurements of muscle oxyhaemoglobin $\left(\mathrm{HbO}_{2}\right)$, deoxyhaemoglobin $(\mathrm{HHb})$ and total haemoglobin $(\mathrm{THb})$ concentration were also made via NIRS (OxiplexTS, ISS, Champaign, USA). Light 
source-detector separation distances of 1.50 - $3.04 \mathrm{~cm}$ for each wavelength were utilised with cell water concentration assumed constant at 70\%. For the present study, data was sampled at $2 \mathrm{~Hz}$. The flexible probe was placed longitudinally along the belly of the left vastus lateralis midway between the greater trochanter and the lateral condyle of the tibia and marked with washable pen such that the probe could be placed in the same position for the second exercise bout. The probe was held firmly in place by elastic Velcro strapping and movement of the optical fibres during cycling limited by taping them to an adjacent table. Following each trial, indentations of the probe on the subject's skin were inspected to confirm that the probe had not moved, which was the case for every exercise transition. The NIRS probe was calibrated prior to each testing session using a calibration block of known absorption and scattering coefficients. Calibration was then cross-checked using a second block of known but distinctly different absorption and scattering coefficients. Each of these procedures was according to the manufacturer's recommendations. Recent research, however, has demonstrated good reproducibility of the NIRS technology used in this study (LeClair et al. 2010).

\section{Data analysis}

Pulmonary oxygen uptake and HHb kinetics were modelled via a single exponential function with a delay relative to the start of exercise using standardised procedures previously used in our laboratory (Marwood et al. 2010). For $\mathrm{p} \dot{V_{2}}$ data, abnormal breaths due to coughs and swallows were first removed from the $\mathrm{p} \dot{V} o_{2}$ data to prevent skewing of the underlying response. The criterion for removal of these breaths was those that were different to the mean of the adjacent four data points by more than three times 
the standard deviation of those four points. Each dataset was interpolated second-bysecond between 0 - $360 \mathrm{~s}$; the two datasets were then ensemble averaged to produce a single response for each subject. The first $20 \mathrm{~s}$ of the ensemble dataset [cardiodynamic phase, (Rossiter et al. 1999) were then removed. The remaining dataset (i.e. up until the end of exercise) was fitted to a mono-exponential curve (Origin, Microcal) with a delay relative to the onset of exercise of the form:

$$
\dot{V} O_{2(t)}=\dot{V} O_{2(b)}+A_{V o_{2}} *\left(1-e^{-\left(t-T D_{V o}\right) / \tau_{V o 2}}\right)
$$

Where $\dot{V}_{o_{2(b)}}$ is the mean $\dot{V}_{o_{2}}$ measured during the final minute of baseline (10 W) exercise, $A_{\mathrm{Vo}_{2}}$ is the asymptotic amplitude of the fundamental (phase II) response, $T D_{\mathrm{Vo}_{2}}$ is a time delay relative to the onset of exercise and $\tau_{V_{o_{2}}}$ is the time constant for the fundamental component of the response. Steady state oxygen uptake ( $\left.\dot{V}_{\mathrm{O}_{(S S)}}\right)$ is therefore the sum of $\dot{V}_{\mathrm{O}_{(b)}}+A_{\mathrm{Vo}_{2}}$ and the mean response time $\left(M R T_{\mathrm{VO}_{2}}\right)$ was defined as $\tau_{\mathrm{Vo}_{2}}+$ $T D_{V o_{2}}$.

The $\mathrm{HHb}$ response to exercise was modelled in a similar fashion to $\dot{\mathrm{V}} \mathrm{O}_{2}$ kinetics; firstly the two datasets for each subject were ensemble averaged to produce a single dataset for each subject with data points at $0.5 \mathrm{~s}$ intervals (i.e. $2 \mathrm{~Hz}$ ). Secondly, the point at which $\mathrm{HHb}$ starts to increase following a short delay of $\sim 5-10 \mathrm{~s}$ (Cooper et al. 1985) was identified. This time-point was identified by fitting two linear regression curves to the first 20 s of the ensemble dataset and, using custom written software and the Solver 
function in Microsft Excel, determining the time-point at which the sum of error squared was minimised. This technique assumes linear characteristics of the data in the first few seconds following the onset of the increase in HHb, which given the short time frame appears reasonable. From this point up until the time at which the $\dot{V}_{2}$ data achieved $98 \%$ of its final value, ( $\mathrm{t}=4 \tau_{\mathrm{Vo}_{2}}+T D_{\mathrm{Vo}_{2}}$, i.e. effective steady state) the data were fitted to a mono-exponential curve (Origin, Microcal) with a delay relative to the onset of exercise of the form:

$$
H H b(t)=H H b_{(b)}+A_{H Н b} *\left(1-e^{\left(t-T D_{н н ь}\right) / \tau_{H н ь}}\right)
$$

Where $\mathrm{HHb}_{(b)}$ is the mean $\mathrm{HHb}$ measured during the final minute of baseline $(10 \mathrm{~W})$ exercise, $A_{H н b}$ is the asymptotic amplitude of the response, $T D_{H н b}$ is a time delay relative to the onset of exercise and $\tau_{\text {ннь }}$ is the time constant for the response. The absolute value of $\mathrm{HHb}$ at $\mathrm{t}=4 \tau_{\mathrm{VO}_{2}}+T D_{V_{o_{2}}}\left(H H b_{(\phi 3)}\right)$ is therefore the sum of $H H b_{(b)}+A_{H H b}$ and the mean response time ( $\left.M R T_{\mathrm{HHb}}\right)$ was defined as $\tau_{\mathrm{HHb}}+. T D_{\text {Hнь }}$. Although it is not certain whether the processes underlying the $\mathrm{HHb}$ response are exponential in nature, visual inspection of the data and reference to previous literature (Cooper et al. 1985; Eriksson et al. 1973; Hughson et al. 1995) suggests that a monoexponential decay model of the form below provides a reasonable estimate of the time course of muscle deoxygenation during this "primary" phase of the $\mathrm{HHb}$ response. The average value during the final $30 \mathrm{~s}$ of exercise $\left(H H b_{(360-330 s)}\right)$ was also calculated, as was the difference between $H H b_{(360-330 s)}$ and $H H b_{(\phi 3)}\left(\boldsymbol{A}_{2 \boldsymbol{H H b}}\right)$. 


\section{Statistical Analysis}

Confidence intervals for $\mathrm{P} \dot{\mathrm{V}}_{2}$ and $\mathrm{HHb}$ were calculated. Inter-group comparisons were made using an independent two-tailed t-test, with homogeneity of variance checked via Levene's test. Data are presented as mean \pm standard deviation and an alpha level of 0.05 was selected. Pearson product moment correlation coefficients were calculated between peak $\dot{V}_{2}$ and the $\dot{V O}_{2}$ time constants for the trained and untrained group. All statistical procedures were carried out using SPSS 17.0 (SPSS, Inc., Chicago, ILL).

\section{Results}

Based upon data derived from the menstrual cycle questionnaire at visit 2; 75\% of the trained adolescent soccer players were in the luteal phase, $13 \%$ were in the follicular phase and $12 \%$ were in the menstrual phase. For the recreationally active adolescents, $43 \%$ were in the luteal phase and $57 \%$ in the follicular phase.

\section{$\mathrm{P} \dot{\mathrm{VO}}_{2}$ kinetics}

Representative plots of both a trained and untrained subject are illustrated in Figure 1. The 95\% confidence intervals for the time constants lay within acceptable boundaries (trained: $3.1 \pm 1.7 \mathrm{~s}$ vs. untrained: $4.6 \pm 1.8 \mathrm{~s}$ ). 
The time constant and mean response time of $\dot{V}_{2}$ kinetics were significantly $(\mathrm{P}<0.05)$ faster in the trained adolescent female soccer players compared to the untrained group (Table 2). The relative magnitude of these differences were a $25 \%$ faster time constant in the trained group compared to the untrained group and a 17\% faster mean response time in the adolescent female soccer players compared to their untrained peers. In order to adjust for the significantly $(\mathrm{P}<0.05)$ higher absolute workload performed by the trained subjects during the square-wave transitions (trained: $105 \pm 24 \mathrm{~W}$ vs. untrained: $74 \pm$ 15W), exercise economy was calculated (Gain); there was no inter-group difference identified for Gain (Table 2). There were no significant correlations between Peak $\dot{V}_{2}$ and time constants for the trained $(r=-.03)$ and untrained groups $(r=0.26)$ respectively.

\section{HHb kinetics}

Representative plots of $\mathrm{HHb}$ kinetics for the two groups are illustrated in Figure 2. The 95\% confidence intervals for the $\mathrm{HHb}$ time constants lay within acceptable boundaries (Trained: $1.56 \pm 0.63$ s vs. Untrained: $2.81 \pm 2.11 \mathrm{~s}$ ). There were no inter-group differences for the time constant $(p=0.07)$ or the mean response time $(p=0.08)$ for $\mathrm{HHb}$ kinetics (Table 3), but both values approached significance. Furthermore, derived values for Cohen's d demonstrated large effect sizes (meaningful physiological differences) for the time constant $(-0.804)$, which was lower in the trained compared to the untrained group and mean response time (-0.777), which was also lower in the trained compared to the untrained group. There were no inter-group differences for time delay, or amplitude. There was no between group difference for $\mathrm{HHb} \Phi$, but the p-value (0.06) approached significance. Using Cohen's d, a medium effect size was generated (0.5017); with the trained group demonstrating a higher value than the untrained (Table 3). 


\section{Discussion}

The major findings of this study were that both the $p \dot{V}_{2}$ time constant (25\%) and the mean response time (17\%) were significantly faster in the trained adolescent female soccer players compared to their recreationally active peers. Furthermore, there was evidence to suggest that this was accompanied by inter-group differences with respect to muscle deoxygenation ( $\mathrm{HHb}$ ) kinetics. Whilst, the p-values did not reach statistical significance ( $\mathrm{p}=0.07$ and 0.08 for the time constant and mean response time, respectively), the strong tendency to do so and the high Cohen's d effect sizes suggest that the difference in the mean values was physiologically significant and therefore indicating that both the $\mathrm{HHb}$ time constant and mean response time were faster in the trained compared to the untrained group.

The $p \dot{V}{ }_{2}$ kinetics data are in line with a large body of both cross-sectional and longitudinal data in adults (Burnley et al. 2006; Fukuoka et al. 2002; Phillips et al. 1995). Furthermore, data from the present study are also in agreement with previous findings from our laboratory (Marwood et al. 2010). In this study, a 25\% faster fundamental phase time constant was identified when comparing trained adolescent male soccer players to a group of recreationally active peers. However, there is a paucity of data characterising $p$ $\dot{V} \mathrm{O}_{2}$ kinetics in trained pre- and post-pubertal females. Data from Winlove et al. (2010) in trained pre-pubertal female swimmers and untrained controls demonstrated fundamental phase time constants similar to those found in the present study (Trained: $25 \mathrm{~s}$ vs. 
Untrained: 37 s) during heavy intensity arm-cranking exercise. Similar time constants were also obtained in pubertal, trained female swimmers and untrained controls performing heavy intensity cycle - ergometry exercise (Trained: 21 s vs. Untrained: 35 s), McNarry et al. (2011). The faster $p \dot{V}_{2}$ time constant seen in the present study in the highly-trained female soccer players therefore extends the findings of McNarry et al. (2011) \& Winlove et al. (2010) to pubertal females with a history of systematic, high intensity, intermittent exercise training associated with training for soccer.

The present data may have implications for aerobic and anaerobic performance and subsequently soccer performance. Evidence exists in the literature, that the $p \dot{V O}_{2}$ time constant derived from moderate domain intensity exercise was related to both the Yo-Yo IR1 (soccer-specific aerobic capacity test-r=0.60) and IR2 (soccer-specific anaerobic capacity tests-r $=0.65)$ in adult male soccer players. These findings suggest that the ability to rapidly activate the aerobic energy systems to support intermittent exercise and reduce the reliance on non-aerobic energy sources may be important in delaying the onset of fatigue and improve soccer performance (Rampinini et al., 2010). Further evidence exists to support this theory (Dupont et al., 2005), as a significant correlation was identified between the time constant of the $p \dot{V}_{2}$ fundamental phase of moderate domain intensity exercise and a relative decrease in time, in a series of repeated, linear speed tests $(\mathrm{r}=0.80)$. Taken together with the present data it would appear that faster $p \dot{V} o_{2}$ kinetics during constant load exercise are reflective of a faster adjustment of $p \dot{V}_{2}$ during repeated sprint exercise and a subsequent maintenance of high-intensity intermittent performance. 
The HHb signal from NIRS represents a balance between the oxygen supply and utilization in the interrogated tissue and may help provide an insight into the mechanisms underpinning the faster $\mathrm{p} \dot{V}_{2}$ kinetics in the trained subjects (Grassi et al. 2003). Scrutiny of the $\mathrm{HHb}$ data in the present study suggests that faster $\mathrm{HHb}$ kinetics may have existed in the trained group since the p-values approached significance for both the time constant and the mean response time and the Cohen's d effect sizes were large suggesting that the differences in the mean values were physiologically significant. This finding suggests that in the present study, enhancements in the mechanisms of $\mathrm{O}_{2}$ utilisation were proportionally more important in speeding the $\dot{V}_{2}$ kinetics than were enhancements of peripheral $\mathrm{O}_{2}$ delivery. This is line with data of McNarry et al. (2011) who also demonstrated faster $\mathrm{HHb}$ kinetics in trained female adolescents (Trained: $12 \mathrm{~s}$ vs. Untrained: $20 \mathrm{~s}$ ) in association with faster $\mathrm{p} \dot{\mathrm{V}} \mathrm{O}_{2}$ kinetics.

There is evidence from the extant literature to support an increase in muscle oxidative enzyme activity (Eriksson et al. 1973; Fournier et al. 1982) with training in children and adolescents. Furthermore, such enhancements to oxidative enzyme activity (citrate synthase and 3-hydroxyacylCoA dehyrogenase) have been shown to occur to a greater extent following 16 weeks (1hr per week) of soccer training in recreationally active women (age 19-47 years) compared to a control group engaged in running training only (Bangsbo et al. 2010) A similar study demonstrated that 12 weeks of recreational football training in an adult population resulted in the conversion of Type IIX to Type IIA muscle fibres which retain more characteristics of Type I, oxidative fibres (Krustrup et al. 2010). Since faster $\mathrm{p} \dot{V}_{2}$ kinetics are associated with enhanced oxidative enzyme activity 
(Phillips et al. 1995) and a greater proportion of Type I fibres (Pringle et al. 2003); these findings indirectly suggest that morphologic and metabolic adaptations that favour aerobic metabolism can be achieved, through soccer training and could have an impact upon reducing the fundamental phase time constant.

In contrast to the present data, our previous study in male adolescents demonstrated similar $\mathrm{HHb}$ kinetics between trained and untrained subjects despite faster $\mathrm{p} \dot{\mathrm{VO}}_{2}$ kinetics in the trained subjects (Marwood et al. 2010). This highlights the possibility of sex differences in the response to training at this age; the ability to deliver and utilize oxygen adapting in equal proportion in males, but mechanisms of oxygen utilization being proportionally more important than oxygen delivery in females.

There were limitations associated with the present study. The cross-sectional nature of the present study can never fully answer the question, as to whether the faster $p \dot{\mathrm{VO}_{2}}$ kinetics is a product of genetic self-selection, maturity-mediated responses or the effect of long-term training adaptations. The trained soccer players in this study were affiliated to professional soccer clubs in the UK and all undertook, high-intensity, intermittent training, as confirmed by the coaches. Therefore, we are confident that faster time constant and mean response time seen in the athletes was as a result of exposure to the training stimulus and not as a result of genetic self-selection. No attempt was made to control the stage of menstrual cycle during testing. It has, however, previously been demonstrated that the menstrual phase has no effect on the $\mathrm{p} \dot{V}_{2}$ or HHb kinetics (Gurd et al. 2007). The sample sizes were small, but were in accord with previous work 
published in this area (McNarry et al. 2011; Winlove et al. 2010). Furthermore, the lack of significant differences in HHb kinetics was offset by the large effect sizes. In the present study, we used cycle ergometry as our model of exercise since whilst not sport specific, it matches the major muscle group used by the players in their sport. Indeed, we have previously shown this mode of exercise to have sufficient discriminatory power to identify differences in $p \dot{V}_{2}$ kinetics between trained adolescent, male soccer players and their untrained counterparts (Marwood et al. 2010).

The present study supports the very limited data that exists that supports the notion that $p \dot{V O O}_{2}$ kinetics are faster in trained adolescent females compared to their recreationally active peers. Specifically, we have shown that high-volume, high-intensity, intermittent soccer training, in pubertal, adolescent females is also reflected by faster $p \dot{V}_{2}$ kinetics. Based upon the NIRS data, and in contrast to our data in male adolescents, the faster kinetics may stem primarily from an enhanced oxygen utilisation capacity in the muscle of the trained females. Therefore it appears that high-volume, high-intensity, intermittent soccer training, results in physiological training adaptations that are unique to adolescent females compared to their male counterparts.

\section{Ethical Standards}

All the experiments conducted in this study comply with the current laws of the United Kingdom.

Conflict of Interests 
The authors declare that there are no conflicts of interest relating to this study. 


\section{References}

Bailey SJ, Wilkerson DP, DiMenna FJ, Jones AM (2009) Influence of repeated sprint training on pulmonary $\mathrm{O}_{2}$ uptake and muscle deoxygenation kinetics in humans. J Appl Physiol 106: 1875-1877. doi: 10.1152/japplphysiol.00144.2009

Bangsbo J, Nielsen JJ, Mohr M, Randers MB, Krustrup BR Brito J, Nybo L, Krustrup P (2010) Performance enhancements and muscle adaptations of a 16-week recreational football intervention for untrained women. Scand J. Med Sci Sports 20: 24-30. doi:10.1111/j.1600-0838.2009.01050.x

Bar-Or, O. Pediatric sports medicine for the practicioner: From physiologic principles to clinical applications. New York: Springer-Verlag, 1983, pp. 376.

Berger NJ, Tolfrey K, Williams AG, Jones AM (2006) Influence of continuous and interval training on oxygen uptake on-kinetics. Med Sci Sports Exerc 38: 504-12. doi: 10.1249/01.mss.0000191418.37709.81

Buchheit M, Mendez-Villanueva A, Simpson BM, Bourdon, PC (2010). Repeated-sprint sequences during youth soccer matches. Int J Sports Med 31: 709-716. doi: 10.1055/s0030-1261897. 
Burnley M, Roberts CL, Thatcher R, Doust JH, Jones AM (2006) Influence of blood donation on $\mathrm{O}_{2}$ uptake on-kinetics, peak $\mathrm{O}_{2}$ uptake and time to exhaustion during severeintensity cycle exercise in humans. Experimental Physiology 91:499-509. doi: 10.1113/expphysiol.2005.032805

Cleuziou C, Lecoq AM, Candau R, Courteix D, Geunon P, Obert P (2002) Kinetics of oxygen uptake at the onset of moderate and heavy exercise in trained and untrained prepubertal children. Sci Sports 17: 291-296. doi: 10.1016/S0765-1597(02)00169-7

Cooper DM, Berry C, Lamarra N, Wasserman K (1985) Kinetics of oxygen uptake and heart rate at onset of exercise in children. J Appl Physiol 59: 211-217. PMID: 3001016

Datson N, Hulton A, Andersson H, Lewis T, Weston M, Drust B, Gregson W (2014) Applied physiology of female soccer: An update. Sports Med 44: 1225-1240. Doi: $10.1007 / \mathrm{s} 40279-014-0199-1$

Demerath EW, Guo SS, Chumlea WC, Towne B, Roche AF, Siervogel RM (2002) Comparison of percent body fat estimates using air displacement plethysmography and hydrodensitometry in adults and children. Int J Obes Relat Metab Disord 26: 389397. doi: 10.1038/sj.ijo.0801898 
Dupont G, Millet GP, Guinhouya C, Berthoin S (2005) Relationship between oxygen uptake kinetics and performance in repeated running sprints. Eur J Appl Physiol 95: 2734. Doi: 10.1007/ s00421-005-1382-8

Eriksson BO, Gollnick PD, Saltin B (1973) Muscle metabolism and enzyme activities after training in boys 11-13 years old. Acta Physiol Scand 87: 485-497.doi: 10.1111/j.1748-1716.1973.tb05415.x

Fawkner S, Armstrong N (2003) Oxygen uptake kinetic response to exercise in children. Sports Medicine 33: 651-659. Dx.doi.org/10.2165/00007256-200333090-00002

Fournier M, Ricci J, Taylor AW, Ferguson RJ, Montpetit RR, Chaitman BR (1982)Skeletal muscle adaptation in adolescent boys-sprint and endurance training and detraining. Med Sci Sports Exerc 14: 453-456. dx. doi.org/10.1249/00005768198214060-00008

Fukuoka Y, Grassi B, Conti M et al (2002) Early effects of exercise training on on- and off-kinetics in 50-year-old subjects. Pflugers Arch 443: 690-7. doi: 10.1007/s00424-001-

0748-y

Gurd BJ, Scheid J, Paterson DH, Kowalchuk JM (2007) Oxygen uptake and muscle deoxygenation kinetics during the transition to moderate-intensity exercise in different phases of the menstrual cycle in young adult females. Eur J Appl Physiol 101: 321-330. doi: 10.1007/s00421-007-0505-9 
Grassi B, Pogliaghi S, Rampichini S, Quaresima V, Ferrari M, Marconi C, Cerretelli P (2003) Muscle oxygenation and pulmonary gas exchange kinetics during cycling exercise on-transitions in humans. J Appl Physiol 95: 149-158. Doi:

10.1152/japplphysiol.00695.2002

Haralambie G (1982) Enzyme activities in skeletal muscle of 13-15 years old adolescents. Bull Europ Physiopathol Resp 18: 65-74. PMID: 7053778

Hughson RL, Kowalchuk JM (1995) Kinetics of oxygen uptake for submaximal exercise in hyperoxia, normoxia, and hypoxia. Can J Appl Physiol 20: 198-210.doi: dx.doi.org/10.1139/h95-014.

International Society for the Advancement in Kinanthropometry. International standards for anthropometric assessment (2001) Underdale SA, Australia: ISAK, , pp. $1-133$.

Jones AM, Koppo K (2005) Effect of training on $\mathrm{VO}_{2}$ kinetics and performance. In: Jones AM, Poole DC (eds) Oxygen uptake kinetics in sport, exercise and medicine. Routledge, London , pp 373-397.

Koppo K, Bouckaert J, Jones AM (2004) Effects of training status and exercise intensity on phase II VO2 kinetics. Med Sci Sports Exerc 36:225-232. doi:

10.1249/01.MSS.0000113473.48220.20 
Krustrup P, Christensen JF, Randers MB, Pedersen H, Sundstrup E, Jakobsen MD, Krustrup B, Nielsen JJ, Suetta C, Nybo L, Bangsbo J (2010) Muscle adaptations and performance enhancements of soccer training for untrained men. Eur J Appl Physiol 108: 1247-1258. doi: 10.1007/s00421-009-1319-8

LeClair E, Borel B, Baquet G, Berthoin S, Mucci P, Thevenet D, Reguem SC (2010) Reproducibility of measurement of muscle deoxygenation in children during exercise. Pediatr Exerc Sci 22:183-194. doi:

Marwood S, Roche D, Rowland T, Garrard M, Unnithan VB (2010) Faster pulmonary oxygen uptake kinetics in trained versus untrained male adolescents. Med Sci Sports Exerc 42: 127-134. doi: 10.1249/MSS.0b013e3181af20d0

Matsudo S, Matsudo V (1994) Self-assessment and physicians assessment of sexual maturation in Brazilian boys and girls: concordance and reproducibility. Am J Hum Biol 6: 451-455. doi: 10.1002/ahjb.1310060406

McNarry MA, Welsman JR, Jones AM (2011) Influence of training status and exercise modality on pulmonary O2 uptake kinetics in pubertal girls. Eur J Appl Physiol 111: 621-631. doi: 10.1007/s00421-010-1681-6 
Obert P, Cleuziou C, Candau R, Courteix D, Lecoq AM, Guenon P (2000) The slow component of $\mathrm{O} 2$ uptake kinetics during high-intensity exercise in trained and untrained prepubertal children. Int J Sports Med 21:31-6. dx.doi.org/10.1055/s-2000-8856

Peterson SR, Gaul CA, Stanton MM, Hanstock CC (1999) Skeletal muscle metabolism during short-term, high-intensity exercise in prepubertal and pubertal girls. J Appl Physiol 87: 2151-2156. doi:

Phillips SM, Green HJ, MacDonald MJ, Hughson RL (1995) Progressive effect of endurance training on VO2 kinetics at the onset of submaximal exercise. J Appl Physiol 79:1914-1920. doi:

Potter CR, Unnithan, VB (2005) Interpretation and implementation of oxygen uptake kinetics studies in children with spastic cerebral palsy. Dev Med Child Neurol 7: 353357. doi: 10.1111/j.1469-8749.2005.tb01148.x

Pringle JS, Doust JH, Carter H, Tolfrey K, Campbell IT, Jones AM (2003) Oxygen uptake kinetics during moderate, heavy and severe intensity submaximal exercise in humans: influence of muscle fibre type and capilliarisation. Eur J Appl Physiol 89 289300. doi: 10.1007/s00421-003-0799-1 
Rampinini E, Sassi A, Azzalin A, Castagna C, Menaspa P, Carlomagno D, Impellizzeri FM (2010) Physiological determinants of Yo-Yo intermittent recovery tests in male soccer players. Eur J Appl Physiol 108: 401-409. Doi 10.1007/s00421-009-1221-4

Rossiter HB, Ward SA, Doyle VL, Howe FA, Griffiths JR, Whipp BJ (1999) Inferences from pulmonary $\mathrm{O}_{2}$ uptake with respect to intramuscular [phosphocreatine] kinetics during moderate exercise in humans. J Physiol 518:921-32. doi: 10.1111/j.14697793.1999.0921p.x

Winlove MA, Jones AM, Welsman JR (2010) Influence of training status and exercise modality on pulmonary oxygen uptakes in pre-pubertal girls. Eur J Appl Physiol 108:169-1179. doi: 10.1007/s00421-009-1320-2 


\section{Figure Captions}

Fig 1 Representative plots of $\mathrm{VO}_{2}$ kinetics for trained (closed squares) and untrained subjects (open squares) with fundamental phase exponential curve fit and residuals shown

Fig 2 Representative plot of HHb kinetics for trained (closed squares) and untrained subjects (open squares) with fundamental phase exponential curve fit and residuals shown 
